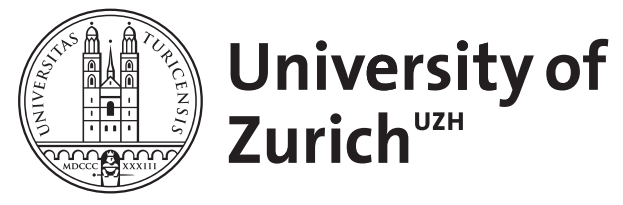

\title{
Urogenitaler Östrogenmangel im Senium
}

\author{
Schiessl, K
}

\begin{abstract}
While urogenital estrogen deficiency has been well investigated in recently perimenopausal or menopausal women, there is a lack of findings referring to elderly women. In addition women avoid discussing symptoms of vaginal atrophy with doctors during consultations. Local estrogen use is the most effective therapy to avoid progressive vaginal atrophy. There is a wide range of nourishing and lubricating preparations if the use of estrogen is to be avoided.
\end{abstract}

DOI: https://doi.org/10.1007/s10304-013-0564-4

Posted at the Zurich Open Repository and Archive, University of Zurich ZORA URL: https://doi.org/10.5167/uzh-90116

Journal Article

Published Version

Originally published at:

Schiessl, K (2013). Urogenitaler Östrogenmangel im Senium. Gynaekologische Endokrinologie, 11(4):243247.

DOI: https://doi.org/10.1007/s10304-013-0564-4 
Gynäkologische Endokrinologie 2013 .

11:243-247

DOI 10.1007/s10304-013-0564-4

Online publiziert: 20. September 2013

๑) Springer-Verlag Berlin Heidelberg 2013

Redaktion

M. Birkhäuser, Basel

K. Schiessl

Klinik für Reproduktions-Endokrinologie, UniversitätsSpital Zürich, Schweiz

Urogenitaler

Östrogenmangel

im Senium

\section{Bedeutung und Vorgehen}

\begin{abstract}
Das Frauenbild der postmenopausalen und älteren Frau ist deutlich im Wandel. Während Elternpflege, Aufzucht der Enkelkinder und die zunehmende eigene Gebrechlichkeit noch vor 1 bis $\mathbf{2}$ Generationen den Aufgaben- und Lebensbereich vieler älterer Frauen darstellten, sind heute auch Seniorenstudium, Reisen, neue Partnerschaften und ein möglichst aktives autonomes Leben wichtige Inhalte.
\end{abstract}

Urogenitale Gesundheit im fortgeschrittenen Alter war bisher vor allem geprägt von der Behandlung dermatologischer Erkrankungen, Präkanzerosen und Malignomen, andererseits der Therapie von Prolapserkrankungen und Harninkontinenz. Heute rücken auch Themen wie Sexualität, Infektionsprophylaxe und allgemeines Wohlbefinden in den Vordergrund und werden von unseren Patientinnen thematisiert. Demgegenüber steht ein fast völliges Fehlen von wissenschaftlich fundierter Erkenntnis. Studien- und Untersuchungsergebnisse beziehen sich überwiegend auf Frauen zwischen 55 und 65 Jahren - lediglich in Bezug auf die Sexualität älterer Menschen liegen zunehmend Daten vor.

\section{Östrogenentzug urogenital}

Der weibliche Urogenitaltrakt reagiert rezeptorvermittelt sehr sensibel auf Östrogene: Hautturgor, Epitheldicke und kolla- genes Bindegewebe, vaginale Bakterienflora und damit der $\mathrm{pH}$ werden davon bestimmt. Wie die Vagina ist die distale Urethra reich an Östrogenrezeptoren und reagiert daher ebenso empfindlich auf Veränderungen des Östrogenspiegels. Betroffene Frauen bemerken östrogenmangelbedingte Beschwerden zum Teil schon in der menopausalen Übergangszeit, am ehesten in Form von zeitweiser vaginaler Trockenheit, zunehmender Dranginkontinenz oder rezidivierenden Harnwegsinfekten. Drei bis 5 Jahre postmenopausal werden die zunehmende Vaginalatrophie und die abnehmende Vaginalsekretmenge bei bis zur Hälfte der Frauen symptomatisch im Sinn von Jucken oder Brennen sowie Missempfindungen, zunehmender Dyspareunie und Kontaktblutungen [2, $6,7,12]$. Mit fortdauerndem Östrogenentzug und zunehmendem Alter verstreichen die Vaginalfalten, und die Vaskularisierung der Schleimhaut geht zurück. Das Vaginalepithel wird dünner und verletzlicher, die Glykogenproduktion nimmt ab. Dadurch sinkt die Besiedelung mit Laktobazillen, und der vaginale $\mathrm{pH}$ steigt vom bisherigen sauren Normalbereich von 3,5-5 auf Bereiche von 6-8 an. Damit besteht zumindest theoretisch ein erhöhtes Infektionsrisiko mit analen oder anderen potenziell pathogenen Keimen, auch wenn sich dies in der vorliegenden Evidenz nicht unbedingt abbildet. Andererseits existieren Hinweise, dass auch eine sekundäre Kolonisierung durch rektale Laktobazillenstämme zur Stabilisierung der Vaginalflora beitragen kann [20]. Bei vaginaler Inspektion finden sich häufig Inflammationszeichen und Petechien, bei mechanischer Irritation kommt es schnell zu oberflächlichen Epithelverletzungen.

Im Gegensatz zu anderen postmenopausalen Symptomen wie Hitzewallungen oder Schlafstörungen nehmen atrophiebedingte vulvovaginale Symptome mit zunehmendem Alter nicht ab. Es kommt zu einer zunehmenden Veränderung der Trophik. Dabei ist es nicht möglich, klar zwischen altersbedingten Veränderungen und Folgen des niedrigen Östrogenspiegels zu unterscheiden.

I) Atrophiebedingte vulvovaginale Symptome nehmen mit zunehmendem Alter nicht ab

Nach Schätzungen westlicher Menopausegesellschaften bemerken $10-50 \%$ der postmenopausalen Frauen Symptome vaginaler Atrophie [2, 3, 6]. Erstaunlicherweise werden trotzdem vulvovaginale Symptome wesentlich seltener im Rahmen einer ärztlichen Konsultation angesprochen, als bei dieser Häufigkeit zu erwarten wäre [2]. Über die Prävalenz speziell bei Frauen höheren Alters liegen keine verlässlichen Daten vor.

Atrophiesymptome betreffen verstärkt Patientinnen nach Karzinomtherapien oder antiöstrogener Therapie mit Aromatasehemmern oder bestimmten se- 
lektiven Östrogenrezeptormodulatoren (SERMs; [22]).

\section{Diagnostik}

Anamnestisch beklagen betroffene Frauen ein vaginales Trockenheitsgefühl, Jucken, eine unangenehme bis schmerzhafte Empfindlichkeit im Urogenitalbereich und Schmerzen beim Geschlechtsverkehr [28]. Die Diagnose einer vermuteten vulvovaginalen Atrophie lässt sich klinisch verifizieren. Die Vulva der älteren Frau zeigt einen Rückgang der Pubesbehaarung und der labialen Fettpolster. Labia majora und minora verstreichen, das Präputium der Klitoris weicht zurück. Eine Spekulumeinstellung zeigt neben einem möglichen Prolaps eher trockenes, dünnes, blasses und leicht vulnerables Epithel, häufig auch eine Verengung des Introitus vaginae. Die Vaginalfalten sind wenig prominent, Durchblutung und Befeuchtung sinken. Im vaginalen Maturationsindex zeigt sich eine deutliche Verschiebung von Superfizialzellen zu intermediären und parabasalen Zellen, häufig besteht eine Mischflora ohne Laktobazillen und vermehrten Leukozyten im Nativpräparat entsprechend einem Nuget-Score 4 [18]. Allerdings korrelieren Beschwerden und Befund nicht stringent [7], Symptome können ohne ausgeprägte Befunde vorhanden sein.

Neu entwickelt liegt jetzt ein validierter Fragebogen zu vulvovaginalen Symptomen vor, der die damit verbundenen Symptome, Affekte und Auswirkungen auf Lebensqualität und Sexualität erfassen soll - eine Thematik, der bislang noch wenig Aufmerksamkeit zukam. Die Testgruppe umfasste 120 ältere, überwiegend kaukasische postmenopausale Frauen in den USA, die im Mittel 66 Jahre alt waren (48 bis 91 Jahre; [7]), und ist damit auch für ältere Frauen ausgelegt.

\section{Differenzialdiagnose}

Dermatologische Erkrankungen sowie Infektionen können ein der vaginalen Atrophie sehr ähnliches Beschwerdebild verursachen. Daher ist zu Beginn jeder Behandlung, mindestens aber bei Versagen einer hormonellen Therapie eine Inspektion des Genitalbereiches zur Diagnostik wesentlich. Auszuschließen sind Lichen sclerosus, Lichen ruber, Pemphigus vulgaris, Pilz- und bakterielle Infektionen sowie eine vorbestehende Vulvadynie, wobei ein Teil dieser Erkrankungen mit vermehrtem Fluor einhergeht.

\section{Urologische Symptomatik}

Die weibliche distale Urethra besitzt wie die distale Vagina eine hohe Dichte an Östrogenrezeptoren, beide entwickeln sich aus dem gleichen embryonalen Gewebe. Daher verändert sich bei fehlendem Östrogeneinfluss auch hier die epitheliale Auskleidung hin zu vermehrten Übergangszellen. Zusammen mit der auch altersbedingten Abnahme der glatten Muskulatur und einer ebenfalls östrogenabhängigen Abnahme der $\alpha$-adrenergen Rezeptoren kommt es zur Absenkung des intraurethralen Verschlussdrucks mit vermehrter Inkontinenzneigung [5].

Pollakisurie, Nykturie, vermehrter Harndrang und Inkontinenz sowie rezidivierende Infektionen nehmen mit dem Alter zu und können die Lebensqualität erheblich beeinflussen. Dabei sind ursächlich Alterserscheinungen, Symptome des Östrogenverlustes und der Effekt zusätzlicher Faktoren wie vorangegangene, evtl. traumatische Geburten, Bindegewebsfestigkeit und zusätzliche Erkrankungen schwer gegeneinander abzugrenzen.

\section{Sexualität}

Der Wunsch von Frauen höheren Alters nach Sexualität ist auch in westlichen Kulturen inzwischen kein Tabuthema mehr. Die Generation, die erstmals von der Möglichkeit einer sicheren und reversiblen Antikonzeption profitieren konnte, entfachte und erlebte die sog. sexuelle Revolution: die Trennung von Sexualleben und Fortpflanzung. Heute bestimmen sie die Normen einer Sexualität im Alter neu [26]. Für mehr als 20\% der Frauen über 75 stellt sie ein wichtiges Element in ihrem Leben dar [15]. Gelebte Sexualität gehört heute zu einem "geglückten Altern“ [29]. Trotzdem fällt es gerade älteren Frauen schwer, von sich aus sexuelle Bedürfnisse und Probleme im Rahmen einer ärztlichen Konsultation anzusprechen [12].
Ein erheblicher Anteil postmenopausaler und älterer Frauen gibt Symptome einer sexuellen Dysfunktion [4] an. Vaginale Trockenheit, Vulnerabilität des Vaginalepithels und Verengung des Introitus können zu deutlicher Beeinträchtigung von sexueller Lust und Befriedigung bei Berührung oder vaginaler Penetration führen. Die Anwendung lokaler hormoneller Therapien oder Gleitmittel kann dem abhelfen $[1,6]$. Dabei darf aber nicht übersehen werden, dass die wesentlichen Faktoren sexueller Aktivität und Zufriedenheit partnerbezogen sind und die weibliche genitale Stimulationsfähigkeit bei adäquaten Stimuli nicht vom Menopausestatus abhängig ist [13]. Einer der Hauptgründe für die Beendigung sexueller Aktivitäten ist für ältere Frauen immer noch das Fehlen oder die Erkrankung des Partners, aber auch der physische und psychische Gesundheitsstatus, soziale Faktoren, Selbstwertgefühl und Körperbild beeinflussen das sexuelle Wohlbefinden wesentlich [15, 17]. Regelmäßige sexuelle Aktivität verbessert die vulvovaginale Durchblutung, Befeuchtung und Elastizität und trägt zur lokalen wie allgemeinen Gesundheit und Lebensqualität bei $[3,27]$.

\section{\) Bei Beschwerden sollte eine genaue Sexualanamnese erhoben werden}

In jedem Fall sollte bei Beschwerden eine genaue Sexualanamnese erhoben werden, um neben möglichen atrophiebedingten Symptomen andere Ursachen eines gestörten Sexuallebens nicht zu übersehen.

\section{Hormonelle Therapie}

\section{Östrogene}

Die Rationale zum Einsatz von Östrogenen besteht in ihrer Fähigkeit, die vaginale Zytologie zu beeinflussen und atrophiebedingte Veränderungen zu verhindern bzw. teilweise rückgängig zu machen [2]. Allerdings ist dies nur bis zu einem bestimmten Umfang möglich. Bei schon weit fortgeschrittenen Veränderungen der Trophik, wie es unbehandelt in höherem 
Alter der Fall sein kann, ist keine substanzielle Änderung mehr zu erwarten.

Standardempfehlung zur Behandlung atropher Vulvovaginalbeschwerden ist die topische Anwendung niedrig dosierter Östrogenpräparate $[1,6]$. Es existiert eine breite Auswahl an Darreichungsformen von Vaginalovula und -zäpfchen über Vaginalcremes, -tabletten bis hin zum hormonsezernierenden Vaginalring. Sie enthalten Östradiol (auch als Acetat oder Hemihydrat), Östriol oder Promestrien als nichtsteroidales Östrogenanalogon. Ihre Wirkung ist im Wesentlichen lokal begrenzt, allerdings werden vor allem zu Therapiebeginn durchaus systemische Spiegel nachgewiesen. Der systemische Effekt ist bei Östradiolpräparaten inklusive dem Vaginalring am stärksten ausgeprägt, während Östriol und das in der Schweiz zugelassene Promestrien eine deutlich niedrigere Systemwirkung zeigen [5]. Aufgrund der guten lokalen Resorption sind schon niedrigste Östrogendosierungen vulvovaginal wirksam und ausreichend [10, 24], dabei sind alle Darreichungsformen effektiv wirksam [28].

\section{》) Schon niedrigste \\ Östrogendosierungen \\ sind vulvovaginal wirksam \\ und ausreichend}

Durch eine rechtzeitig und konsequent angewandte Östrogentherapie steigt der vaginale $\mathrm{pH}$, das Epithel nimmt an Dicke zu, die Durchblutung des Gewebes steigt an, und die Lubrifikation wird verbessert. Die vaginale Trockenheit und eine dadurch bedingte Dyspareunie nehmen ab, die Trophik verbessert sich, die Vulnerabilität geht zurück.

Auf eine lokale oder systemische Zugabe von Gestagenen oder Progesteron kann bei diesen niedrig dosierten Präparaten verzichtet werden, entsprechende Untersuchungen zur endometrialen Sicherheit umfassen allerdings nur Zeiträume bis zu 1 Jahr [1]. Bei dem Auftreten einer vaginalen Blutung ist eine endometriale Abklärung indiziert.

Klassisch beginnt die vaginale Östrogentherapie mit einer Aufsättigungsphase, in der über 2 bis 3 Wochen eine tägliche Anwendung erfolgt. Anschließend ist im
Allgemeinen eine 1- bis 2-mal wöchentliche Anwendung ausreichend, um den Effekt zu erhalten. Der 2 mg Östradiol enthaltende Vaginalring wird über 3 Monate intravaginal belassen und gibt im Mittel $7,5 \mu \mathrm{g} / 24 \mathrm{~h}$ ab. Eine Dosiserhöhung kann bei allen Präparationen zu systemischen Reaktionen führen und endometrial wirksam sein.

Eine systemische menopausale Hormontherapie ist ebenfalls vaginal wirksam, allerdings bezüglich der Atrophiesymptome nicht von Vorteil gegenüber der topischen Therapie und allein in dieser Indikation daher nicht indiziert. Andererseits zeigt eine niedrig dosierte vaginale Östrogentherapie keinen Effekt auf vasomotorische Symptome und vermindert das Risiko einer osteoporotischen Fraktur nicht [2].

Bei Zeichen der Dranginkontinenz oder rezidivierenden Harnwegsinfekten sollte der Einsatz einer lokalen Östrogentherapie erwogen werden $[3,6]$, auch wenn der positive Effekt weniger eindeutig ist als bei vaginalen Atrophiesymptomen. Das ist nicht zuletzt in der komplexen Struktur der ursächlichen Faktoren begründet. Eine vorbestehende Harninkontinenz wird nach den vorliegenden Daten der WHI- und HERS-Studie durch eine systemische Östrogentherapie eher verschlechtert $[8,9]$.

\section{Ospemifen}

Neu ist in den Vereinigten Staaten Ospemifen, ein selektiver Östrogen-Rezeptor-Modulator (SERM) für die Behandlung der atrophiebedingten mittleren und schweren Dyspareunie zugelassen [6]. Das dem Tamoxifen strukturähnliche Ospemifen wird oral eingenommen und wirkt am Vaginalepithel östrogen, ist allerdings auch am Endometrium agonistisch wirksam. Auf vasomotorische Beschwerden wirkt es verstärkend [23]. Ob es wie andere SERMs gleichzeitig osteound mammaprotektive Eigenschaften besitzt, ist noch in Studien zu eruieren. Bisher existieren lediglich präklinische Daten [21].
Gynäkologische

Endokrinologie 2013 $\cdot 11: 243-247$

DOI 10.1007/s10304-013-0564-4

(c) Springer-Verlag Berlin Heidelberg 2013

\section{K. Schiessl \\ Urogenitaler Östrogenmangel im Senium. Bedeutung und Vorgehen}

\section{Zusammenfassung}

Während der urogenitale Östrogenmangel bei jüngeren oder peri- bis postmenopausalen Frauen bis 55 Jahre gut untersucht ist, fehlen eigenständige Daten für das höhere Frauenalter weitgehend. Zusätzlich werden entsprechende Symptome von den betroffenen Frauen zu selten im Rahmen ärztlicher Konsultationen angesprochen. Eine topische Östrogentherapie ist die bisher wirksamste Behandlung zur Vermeidung einer fortschreitenden Atrophisierung. Es existiert eine breite Palette zusätzlicher pflegender und befeuchtend wirksamer Substanzen, wenn eine Östrogenbehandlung vermieden werden soll.

\section{Schlüsselwörter}

Urogenitale Atrophie im Senium .

Dyspareunie · Vaginale Östrogentherapie .

Vaginale Trockenheit · Sexualität bei Älteren

\section{Urogenital estrogen deficiency in elderly women. Importance and approach}

\section{Abstract}

While urogenital estrogen deficiency has been well investigated in recently perimenopausal or menopausal women, there is a lack of findings referring to elderly women. In addition women avoid discussing symptoms of vaginal atrophy with doctors during consultations. Local estrogen use is the most effective therapy to avoid progressive vaginal atrophy. There is a wide range of nourishing and lubricating preparations if the use of estrogen is to be avoided.

\section{Keywords}

Urogenital atrophy elderly - Dyspareunia . Vaginal estrogen therapy - Vaginal dryness . Sexuality elderly

\section{DHEA}

Dehydroepiandrosteron (DHEA) wird gut von der Vaginalschleimhaut resorbiert und verbessert - topisch als Creme täglich angewandt - den Maturationsindex, senkt den vaginalen $\mathrm{pH}$ und scheint die Dyspareunie zu vermindern. Da bis- 
her kein DHEA-Rezeptor bekannt ist, beruht diese Wirkung am ehesten auf metabolischer Umwandlung in Östron bzw. Testosteron und Östradiol. Bisher liegen allerdings kaum unabhängige randomisiert kontrollierte Daten zur Anwendung vor [6]. DHEA zeigt in den bisher erprobten Dosierungen keine östrogenen Effekte auf das Endometrium, und die Serumwerte von Östradiol oder Testosteron bleiben im Rahmen postmenopausaler Normwerte [19]. Bisher ist kein entsprechendes Präparat in den deutschsprachigen Ländern zugelassen.

\section{Testosteron}

Die tägliche intravaginale Applikation von Testosteron mit und ohne Östrogene zeigt eine signifikante Verbesserung von vulvovaginalen Atrophiesymptomen und scheint sich positiv auf die Verbesserung der Sexualität von Frauen mit sexueller Dysfunktion auszuwirken [19]. Hinter Letzterem ist allerdings ein systemischer Effekt zu vermuten. Bisher existieren kaum unabhängige Untersuchungen, es liegen keine Langzeitdaten zu dieser Therapieform vor. Die Dichte der vulvovaginalen Androgenrezeptoren ist möglicherweise östrogenabhängig [5], was für eine kombinierte Anwendung spräche.

\section{Steroidbehandlung nach hormonabhängigen Tumoren}

Für die Behandlung von Frauen nach hormonabhängigen gynäkologischen Malignomen ist die topische Östrogenanwendung nicht in randomisiert kontrollierten Studien untersucht und gilt daher weiterhin als nicht ausreichend gesichert. Unkontrollierte, kleine Beobachtungsstudien zeigten keine erhöhte Rezidivhäufigkeit bei Mammakarzinompatientinnen [25], die Wirkung eingesetzter Aromataseinhibitoren scheint nicht beeinträchtigt [19]. Ob Testosteron, Oxytocin [11] oder DHEA Ausweichmöglichkeiten darstellen, ist nicht ausreichend untersucht [6].

\section{Phytoöstrogene}

Isoflavone sind strukturell verwandt mit Östrogenen und können am Östrogen- rezeptor binden, wobei sie analog, aber auch antiöstrogen wirksam sind. Studiendaten zeigen für topisch angewandtes Genistein eine Verbesserung genitaler Atrophiesymptome [14]. Eine vermehrte Isoflavonaufnahme mit der Nahrung führte hingegen bei peri- und postmenopausalen Thailänderinnen nicht zu einer entsprechenden Verbesserung [16], ein Befund, der den meisten vorausgegangenen Untersuchungen entspricht. Für die Sicherheit der Anwendung bei Frauen mit hormonabhängigen Tumoren liegen bisher keine ausreichenden Daten vor [5].

\section{Topische nichthormonelle Therapie}

Der Abbruch der WHI-Studie bzw. die stark medial aufgegriffenen Ergebnisse haben zu einer starken Verunsicherung postmenopausaler Frauen in Bezug auf hormonelle Therapien geführt. Mögliche Risiken und Warnhinweise können von ihnen nicht differenziert werden und werden damit als allgemeingültig verstanden. In Sorge, dass hormonelle Therapien grundsätzlich ein erhöhtes Gesundheitsrisiko beinhalten, stehen diese Frauen einer lokalen Östrogentherapie kritisch gegenüber und favorisieren andere Therapiemöglichkeiten [12]. Auch Frauen mit und nach Mammakarzinom stellt sich die Frage nach Alternativen, solange keine Studien mit entsprechendem Evidenzniveau die Sicherheit der vaginalen, niedrig dosierten Anwendung belegen. Gerade sie sind durch iatrogene Menopause und nachfolgende antiöstrogene Therapien anhaltend und stark von Symptomen des Östrogenentzuges betroffen. Als Alternative steht eine breite Palette von Feuchtigkeits- und Fettcremes sowie Gleitmitteln zur Verfügung. Die meisten Produkte sind rezeptfrei erhältlich. Dabei sollte die Basisbehandlung immer in einer pflegenden fettreichen Creme oder öligen Lotion zum Schutz der Hautbarriere bestehen, gerade bei erhöhter Beanspruchung im Rahmen einer Harninkontinenz.

Befeuchtungsmittel auf Polymerbasis heften sich an die Vaginalwand und binden als hydrophile Substanzen Wasser. Damit steigen intravaginale Feuchtigkeit und Elastizität für einen Zeitraum von 1 bis 5 Tagen, ohne dass der zelluläre Maturationsindex verändert wird. Durch ihren niedrigen $\mathrm{pH}$ puffern sie das basische Vaginalmilieu. Da sie spätestens mit dem physiologischen Turnover der epithelialen Zellen eliminiert werden, ist eine regelmäßige - mehrmals wöchentliche - Anwendung nötig. Entsprechend angewandt, können sie Jucken, Vulvodynie, Vulnerabilität und Dyspareunie deutlich lindern oder beheben auf ein Niveau, das subjektiv dem Erfolg einer topischen Therapie mit einer östrogenhaltigen Creme entspricht $[3,25]$. Auf die zelluläre oder Bindegewebsstruktur haben sie keinen wesentlichen Einfluss. Teilweise enthalten sie zusätzlich wundheilende Pflanzenextrakte, Öle, Phytoöstrogene und Vitamin E. Befeuchtungsmittel auf Pectinoder Hyaluronsäurebasis sind vergleichbar wirksam ohne störenden Ausfluss. Alle Befeuchtungsmittel verbessern die Lubrifikation.

Gleitmittel dienen eher dem kurzfristigen Einsatz beim Geschlechtsverkehr. Sie basieren auf wasser- oder silikonhaltigen Formulierungen, enthalten häufig Glycerin oder Parabene und können zu Reizungen aufgrund höherer Osmolarität führen. Sie können ebenfalls pflanzliche Extrakte oder Phytoöstrogene enthalten und sind im Allgemeinen frei verkäuflich [25]. Gleitmittel haben keine längerfristig positiven Auswirkungen auf atrophiebedingte Symptome, helfen aber bei Dyspareunie und möglicher Irritation des vulnerablen Gewebes.

Laktobazillen stellen prämenopausal das saure Vaginalmilieu durch Milchsäureproduktion sicher und beugen Infektionen vor. Postmenopausal sinkt die Besiedelung mit Laktobazillen und der Vaginal-pH steigt an. Ob die Beigabe von Laktobazillen $\mathrm{zu}$ den verschiedenen Präparationen einen zusätzlichen Effekt auf die Verhütung von Infektionen hat, ist bisher nicht bewiesen.

\section{Vitamine}

Eine türkische Arbeitsgruppe konnte anhand einer kleinen Patientinnengruppe einen Einfluss von Vitamin D auf das Vaginalepithel zeigen, allerdings fehlen weitere klinische Daten in Bezug auf Symptome der vulvovaginalen Atrophie. Vitamin E kann laut Studiendaten die Lubrifi- 
kation verbessern [5] und wird als zusätzlicher Wirkstoff in Gleitmitteln eingesetzt.

\section{Fazit für die Praxis}

- Urogenitale und sexuelle Gesundheit sollten routinemäßig, aber sensibel im ärztlichen Gespräch thematisiert werden.

- Eine topische Östrogenanwendung ist die Standardtherapie bei urogenitalen Atrophiebeschwerden und ist der systemischen zur Besserung vulvovaginaler Symptome überlegen.

- Eine topische Östrogenisierung sollte bei Beschwerden möglichst früh implementiert werden, um ein Fortschreiten zu vermeiden.

- Eine erfüllende und befriedigende Sexualität ist hilfreich, um Befeuchtung und Elastizität zu erhalten.

\section{Korrespondenzadresse}

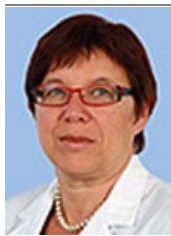

Dr. K. Schiessl

Klinik für Reproduktions-

Endokrinologie,

UniversitätsSpital Zürich

Rämistr. 100, 8091 Zürich

Schweiz

katharina.schiessl@usz.ch

\section{Einhaltung ethischer Richtlinien}

Interessenkonflikt. K. Schiessl war in Beraterboards der Firmen Pfizer, Vifour, Daijichi Sankyo, Amgen und Bayer und erhielt Vortragshonorar bzw. Autorenhonorar von den Firmen Kade und Abbott.

Dieser Beitrag beinhaltet keine Studien an Menschen oder Tieren.

\section{Literatur}

1. Anonymous (2012) The 2012 hormone therapy position statement of The North American Menopause Society. Menopause 19:257-271

2. Anonymous (2007) The role of local vaginal estrogen for treatment of vaginal atrophy in postmenopausal women: 2007 position statement of The North American Menopause Society. Menopause 14:355-369 (quiz 370-351)

3. Anonymous (2005) SOGC clinical practice guidelines. The detection and management of vaginal atrophy. Number 145, May 2004. Int J Gynaecol Obstet 88:222-228

4. Basson R, Leiblum S, Brotto L et al (2004) Revised definitions of women's sexual dysfunction. J Sex Med 1:40-48
5. Birkhäuser M (Hrsg) (2013) Präventionskonzepte und aktuelle Therapieempfehlungen in der Periund Postmenopause. UNI-MED-Verlag AG, Bremen Boston London

6. De Villiers TJ, Pines A, Panay N et al (2013) Updated 2013 International Menopause Society recommendations on menopausal hormone therapy and preventive strategies for midlife health. Climacteric 16:316-337

7. Erekson EA, Yip SO, Wedderburn TS et al (2013) The Vulvovaginal Symptoms Questionnaire: a questionnaire for measuring vulvovaginal symptoms in postmenopausal women. Menopause 20:973-979

8. Grady D, Brown JS, Vittinghoff E et al (2001) Postmenopausal hormones and incontinence: the $\mathrm{He}$ art and Estrogen/Progestin Replacement Study. Obstet Gynecol 97:116-120

9. Hendrix SL, Cochrane BB, Nygaard IE et al (2005) Effects of estrogen with and without progestin on urinary incontinence. JAMA 293:935-948

10. Jaisamrarn U, Triratanachat $S$, Chaikittisilpa $S$ et al (2013) Ultra-low-dose estriol and lactobacilli in the local treatment of postmenopausal vaginal atrophy. Climacteric 16:347-355

11. Jonasson AF, Edwall L, Uvnas-Moberg K (2011) Topical oxytocin reverses vaginal atrophy in postmenopausal women: a double-blind randomized pilot study. Menopause Int 17:120-125

12. Kingsberg SA, Krychman ML (2013) Resistance and barriers to local estrogen therapy in women with atrophic vaginitis. J Sex Med 10:1567-1574

13. Laan E, Van Driel EM, Van Lunsen RH (2008) Genital responsiveness in healthy women with and without sexual arousal disorder. J Sex Med 5:14241435

14. Le Donne M, Caruso C, Mancuso A et al (2011) The effect of vaginally administered genistein in comparison with hyaluronic acid on atrophic epithelium in postmenopause. Arch Gynecol Obstet 283:1319-1323

15. Lochlainn MN, Kenny RA (2013) Sexual activity and aging. J Am Med Dir Assoc 14:565-572

16. Manonai J, Songchitsomboon S, Chanda Ket al (2006) The effect of a soy-rich diet on urogenital atrophy: a randomized, cross-over trial. Maturitas 54:135-140

17. Montemurro B, Gillen MM (2013) Wrinkles and sagging flesh: exploring transformations in women's sexual body image. J Women Aging 25:3-23

18. Nugent RP, Krohn MA, Hillier SL (1991) Reliability of diagnosing bacterial vaginosis is improved by a standardized method of gram stain interpretation. J Clin Microbiol 29:297-301

19. Panjari M, Davis SR (2011) Vaginal DHEA to treat menopause related atrophy: a review of the evidence. Maturitas 70:22-25

20. Petricevic L, Domig KJ, Nierscher FJ et al (2013) Differences in the vaginal lactobacilli of postmenopausal women and influence of rectal lactobacilli. Climacteric 16:356-361

21. Pines A (2013) SERMs, vulvovaginal atrophy and breast cancer. Climacteric 16:398-399

22. Pinkerton JV, Stanczyk FZ (2013) Clinical effects of selective estrogen receptor modulators on vulvar and vaginal atrophy. Menopause [Epub ahead of print]

23. Portman DJ, Bachmann GA, Simon JA (2013) Ospemifene, a novel selective estrogen receptor modulator for treating dyspareunia associated with postmenopausal vulvar and vaginal atrophy. Menopause 20:623-630
24. Simon JA, Maamari RV (2013) Ultra-low-dose vaginal estrogen tablets for the treatment of postmenopausal vaginal atrophy. Climacteric 16(Suppl 1):37-43

25. Sinha A, Ewies AA (2013) Non-hormonal topical treatment of vulvovaginal atrophy: an up-to-date overview. Climacteric 16:305-312

26. Stones M, Stones L (2007) Sexuality, sensuality, and intimacy. In: Birren JE (Hrsg) Encyclopedia of gerontology: age, aging and the aged. Elsevier/ Academic Press, Oxford, S 482-489

27. Sturdee DW, Panay N (2010) Recommendations for the management of postmenopausal vaginal atrophy. Climacteric 13:509-522

28. Suckling J, Lethaby A, Kennedy R (2006) Local oestrogen for vaginal atrophy in postmenopausal women. Cochrane Database Syst Rev:CD001500

29. Wood A, Runciman R, Wylie KR et al (2012) An update on female sexual function and dysfunction in old age and its relevance to old age psychiatry. Aging Dis 3:373-384 